\title{
Time-dependent Network Algorithm for Ranking in Sports
}

\author{
András London, József Németh, and Tamás Németh*
}

\begin{abstract}
In this paper a novel ranking method which may be useful in sports like tennis, table tennis or American football, etc. is introduced and analyzed. In order to rank the players or teams, a time-dependent PageRank based method is applied on the directed and weighted graph representing game results in a sport competition. The method was examined on the results of the table tennis competition of enthusiastic sport-loving researchers of the Institute of Informatics at the University of Szeged. The results of our method were compared by several popular ranking techniques. We observed that our approach works well in general and it has a good predictive power.
\end{abstract}

Keywords: Colley method, Least squares method, Keener method, Markov chain, PageRank, ranking algorithms, self-organization

\section{Introduction}

In the last decade, rating and ranking methods have been studied and applied in a wide range of different areas. Due to the extraordinary success of Google's PageRank (PR) algorithm [7] -originally developed for ranking webpages based on their importance- graph based algorithms have gained more ground in the topic of ranking problems. Some good surveys on the PageRank method can be found in [4, 20, 29]. Recently, the dynamic extensions of the PageRank method have also been discussed, containing the dynamic aspects of the 'damping' factor [28] and the viewpoint of the evolving network [3] and the time dependency [2]. More recently, a novel dynamic ranking model has also been proposed for ranking in sports [23].

Ranking athletes in individual sports, or sport teams is important for those who are interested in the various professional or amateur leagues as a financial investor, a manager or a fan and it also has a crucial role in sports betting from the point of view of both the better and the betting agency. In many sports, only the win/loss ratio is considered (e.g. see the most popular sports in the U.S.) for ranking, i.e. higher value indicates higher position in the ranking. In the case of equal win/loss rates, the result(s) of the head-to-head matches

\footnotetext{
${ }^{*}$ University of Szeged, Department of Computer Science
}

E-mail: \{london, nemjozs, tnemeth\}@inf.u-szeged.hu 
between the players/teams in question and other simple statistics are considered to determine the ranking positions. In many sports, instead of the round-robin system, the type of the most relevant competitions is a single-elimination tournament (also called knock-out or cup) maybe with a preceding group stage. Thus the players play just few matches against only a small subset of the competitors. The official ranking of the players is usually determined by a sport specific rating system (e.g. see tennis, table tennis, combat sports, etc.). In fact, in a tournament, in a regular season or in a given period each player/team plays with only a subset of the others and a player/team who plays against weaker opponents have a considerable advantage compared with those, who play against stronger ones.

Many approach traces back the ranking problem to the solution of a system of linear equations, where the entries of the coefficient matrix refer in some way to the results of the games have been played. Due to the study of this pairwise comparison scheme (for early studies see e.g. $[6,12,19])$, several matrix-based ranking algorithm have been appeared related to the ranking in sports (see e.g. [10] for chess teams, [11, 26] for tennis players, $[5,8,14,21]$ for American football teams). For a good mathematical guide to sports, see e.g. [16], while a useful comprehensive work can be found in [13] and [21].

In this paper, we continue this direction of studies and present a simple, time-dependent PageRank based method, the time-dependent PageRank (tdPR), and apply it to the table tennis competition of the Institute of Informatics at the University of Szeged. In that competition, there is no any regular organization rule: players play against any participant whenever they want. Not even the number of winning sets needed for a win is stated. The only restriction is that 7 days must be elapsed between two matches against the same players. One of the biggest advantage of using this data set is its similarity to the result database of many professional sports in a given period, due to the large variety of the number of matches between two players and the elapsed time between the matches. However most of the (professional) sports have strong conditions for the opponent selection and the number of matches. It can be assumed that without knowing the organizational rules, the 'opponent selection' in a given period can be regarded as a random process (we note that this is not hold e.g. for a Swiss-system chess tournament). Furthermore, we think that the importance of a certain result is inversely proportional to that how old that game is. Thus considering this time-dependency (i.e. , the latest results are more important than the older ones) helps to get clearer picture about the actual relative strengths of the opponents.

Results presented here were compared to other traditional and widely used ranking methods. We highlight the advantages of the usage of our method and show its higher predictive power than the other methods. Furthermore, we also suggest a deeper study of a self-organization mechanism respect to the opponent selection: the players having similar tdPR values more likely to play with each other in the later part of the competition (without knowing the scores and ranks of each other). This observation can explain the appearance of different strength classes and emergence of the elite in several sports.

This paper is organized as follows: in Section 2, we give a brief mathematical description of the methods we used and compared, in Section 3, we apply the methods to the table tennis competition and highlight the usefulness and advantages of our approach. finally, in Section 4, we suggest a new type of a self-organization mechanism and a type of graph regularity for deeper analysis. 


\section{Overview of the ranking methods}

In this section we introduce some widely used ranking methods and describe the proposed approach. Hundreds of ranking methods have been appeared in the long history of ranking in sports. The selection of the methods we use in this paper based on a few criterion: (1) the method is based on linear algebra, (2) the method has been proved to be successful for real applications, and (3) the method has simple formulation with closed form solution.

In this section the number of players is denoted by $N$ while we will refer to the players by $1, \ldots, N$.

\subsection{Least squares method}

The first method we describe is usually referred as the least squares (or weighted least squares) method (Lsm) originated from Smith and Gulliksen [15, 30]. Kenneth Massey in his master thesis found wonderful applications of it, especially for ranking the collage football teams in the United States ([21], Chapter 4). The only statistics used by this method are the number of wins and losses of each player. The ranking of the players comes from the solution of the linear system of equations

$$
M \vec{r}=\vec{p},
$$

where $\vec{r}=\left(r_{1}, \ldots, r_{N}\right)$ is the unknown rating vector of the players, $\vec{p}=\left(p_{1}, \ldots, p_{N}\right)$ the vector contains the difference of total number of wins and losses for the players, while $M$ (we call it Massey matrix) defined as

$$
M_{i j}= \begin{cases}n_{i}, & \text { if } \quad i=j \\ -n_{i j}, & \text { if } \quad i \neq j\end{cases}
$$

where $n_{i}$ is the total number of matches played by player $i$ and $n_{i j}$ is the number of matches played between player $i$ and player $j$. Since $\operatorname{rank}(M)=N-1$, the linear system Eq. (1) is underdetermined. The non-singularity can be guaranteed if each element of any row $i$ of $M$ is set to 1 and the corresponding $p_{i}$ is set to 0 . Obviously, the decreasing order of the components of the rating vector $\vec{r}$ gives the ranking of the players.

\subsection{Colley matrix method}

The Colley matrix mehod was designed by Wesley N. Colley [8]. The method is a modification of the Least squares method by using an observation called Laplace's rule of succession (see [27], page 148) which claims, that if one observed $k$ successes out of $n$ attempts, then $(k+1) /(n+1)$ is better estimation for the next event to be success than $k / n$. The rating vector $\vec{r}$ of the players is the solution of the linear system

$$
C \vec{r}=\vec{b}
$$

where the $i$ th component of the vector $\vec{b}$ is defined as $b_{i}=1+\left(w_{i}-l_{i}\right) / 2$, where $w_{i}$ and $l_{i}$ are the number of wins and losses of player $i$, respectively, and the Colley matrix $C$ is 
defined as

$$
C_{i j}=\left\{\begin{array}{lll}
n_{i}+2, & \text { if } \quad i=j, \\
-n_{i j}, & \text { if } \quad i \neq j .
\end{array}\right.
$$

Thus $C=M+2 I$, where $I$ is the $N \times N$ identity matrix. It can be checked that system Eq. (3) always has a unique solution and just as before, the ranking of the players is obtained by the vector $\vec{r}$

\subsection{Keener method}

James P. Keener developed his ranking method [17], based on the theorem of Frobenius and Perron (see e.g. [22], Chapter 8). Using this method, the ranking of the players comes from the eigenvalue equation

$$
K \vec{r}=\lambda \vec{r},
$$

where the Kenner matrix $K$ defined as

$$
K_{i j}= \begin{cases}\frac{w_{i j}+1}{n_{i j}+2}, & \text { if player } i \text { played against player } j, \\ 0, & \text { otherwise }\end{cases}
$$

where $w_{i j}$ is the number of wins of player $i$ against player $j$ while $\lambda$ is the dominant eigenvalue (the eigenvalue of the largest absolute value, also known as the spectral radius) of the matrix $K$. The Frobenius-Perron theorem guarantees the existence and uniqueness of the vector $\vec{r}$ with strictly positive components. We mention, that the method has been originally defined for ranking American football teams and used the concrete points that a team $i$ scored against a team $j$ and also used a smoothing function to avoid the possibilities for manipulation. For the table tennis competition that has been examined in this paper, we do not deal with the points scored in the games played just consider the final result of each game as win or loss.

\subsection{PageRank method}

The PageRank algorithm - developed by Sergey Brin and Larry Page [7] - was originally designed to rank web pages in order to their importance. The idea behind the algorithm came from the basic properties of Markov chains (see e.g in [27], Chapter 4) as a special case of the Frobenius-Perron theory. The ranking points of the players are iteratively calculated by the recursion formula

$$
P R(i)=\frac{\lambda}{N}+(1-\lambda) \sum_{j \in N^{+}(i)} \frac{P R(j)}{w_{j}},
$$

where $N^{+}(j)$ is the set of players defeated by player $i$ at least once, $w_{j}$ is the total number of wins of player $j$ and $\lambda \in[0,1]$ is a free parameter (usually 0.1 or 0.2 ; the intuitive meaning of $\lambda$ is described in Section 2.5).

To see the close relationship between PageRank formula and the theory of Markov chains, we write Eq. (7) to the vector equation form

$$
\overrightarrow{P R}=\frac{\lambda}{N}\left[I-(1-\lambda) A D^{-1}\right]^{-1} \overrightarrow{\mathbb{1}},
$$


where $\overrightarrow{P R}$ PageRank vector contains the PageRank rates of the players, $A$ is the matrix with elements $A_{i j}$ equals to the number of wins of player $i$ against player $j, D$ the diagonal matrix such that $\left.D=\operatorname{diag}\left[\left(D_{i i}=\sum_{\ell=1}^{N} A_{i \ell}\right)_{i=1}^{N}\right)\right], I$ is the $N \times N$ identity matrix and finally $\overrightarrow{\mathbb{1}}$ is the $N$-dimensional vector having each component equals to 1 . Assuming that $\overrightarrow{\mathbb{P}} \overrightarrow{P R}=1$, Eq. (8) implies, that

$$
\overrightarrow{P R}=M \overrightarrow{P R}
$$

with $M=\lambda / n \overrightarrow{\mathbb{1}} \overrightarrow{\mathbb{1}}^{T}-(1-\lambda) A D^{-1}$, which shows that $\overrightarrow{P R}$ is the eigenvector of the matrix $M$ due to the eigenvalue 1 , which is the largest eigenvalue of $M$ by a consequence of the Frobenius-Perron theorem for row-stochastic matrices.

\subsection{Time-dependent PageRank method}

Intuitively, the basic PageRank algorithm can be considered as a random walk in the graph $G=(V, E)$, where $V$ denotes the set of players and we draw a directed edge $i \rightarrow j \in E$ each time when player $i$ wins against player $j$. The walk starts in a random node $i$ of the graph and steps to a randomly chosen node $j$, with uniform probability, for that $i \rightarrow j$ edge exits. The parameter $\lambda$ can be viewed as a "damping" factor which guarantee that the random walk restarts in a random, uniformly chosen node of the graph almost surely in every $1 / \lambda$ th step. The PageRank of a node $i$ can be considered as the the long-term fraction of the number of visits in node $i$ during the random walk.

Following this direction, we modified the PageRank algorithm such that the weight (i.e the transition probability) of each edge decreases whenever a new edge appears in the graph. Formally, after the $k$ th match was played in a given period, the weight of the latest edge become 1 , the second latest become $1 / 2$, the $i$ th latest become $1 / i$, the oldest one become $1 / k$. We normalize the weights such that the obtained matrix become rowstochastic (i.e. each row summing to 1 ) and we recalculate the ranking every time when a new result is registered in the database by solving the equation

$$
\overrightarrow{P R}=M^{\prime} \overrightarrow{P R}
$$

where the entries of $M^{\prime}$ are then the new transition probability values, calculated as we described.

\section{Experimental results}

We applied the methods described above to the table tennis competition of the Institute of Informatics at the University of Szeged (the dataset we used can be found in the website [1]). In that competition, there is no any rule for the selection of the opponents or the date of the match. The only restriction is that 7 days must be elapsed between two matches of the same players. Without considering the organizational rules and by just considering the list of the results in a given period, it can be observed, that these features are occurred in many sports where the competitions are not round-robin.

In Table 1, we report the scores of the players obtained by the different ranking methods. In the case of the PR and the time tdPR algorithms, we used $\lambda=0.1,0.2,0.3,0.4$, 
respectively. Figure 3 shows, that the tdPR score is very robust against these variations of $\lambda$. The tdPR method was proved to be very effective in finding the best players of the competition that could be a posteriori justified by knowing the players skills.

We used Kendall's $\tau$ rank correlation method [18] to quantify the rank correlation between the different methods. The rank correlation coefficient is defined as $\tau=\left(n_{c}-\right.$ $\left.n_{d}\right) /\left(\begin{array}{l}n \\ 2\end{array}\right)$, where $n_{c}\left(n_{d}\right)$ is the number of such pairs that have the same (opposite) order in both ranking list. However, the tdPR score is positively correlated with the win ratio, differences can be seen by comparing the two methods. The relation between the tdPR and the winning ratio is shown in Figure 1(a).

A relevant outlier on the list is player 14 having win ratio $50 \%$, who precedes player 5, 23, 19 and 21 having better win ratio than himself. He is placed at position 4 and this is consistent with the fact, that he was defeated by just that players (player 10, player 12; see the data set and Figure 4) who ranked higher. Figure 1(b) shows the relation between tdPR and the other ranking methods.

Despite the high correlation between tdPR and the other methods, we observed, that the time-dependent method has a better predictive power. We considered the first half of the total number matches had been played since the start of the competition and calculate the tdPR values regarding that period. Then we checked the results of the upcoming matches and the changes in the ranking. It can be observed, that the players with much higher tdPR score after the first half the total matches played, won a high proportion of their matches against players with smaller tdPR values in the later part of the competition. The difference between the tdPR values of the players can give a reliable prediction for the upcoming matches. Figure 2 shows the tdPR ranks of the players after 45, 90 and 180 played games. We mention, that Figure 2 only contains that players, who had already had at least one played matches after the first 45 played matches of the competition. Obviously, at that time we can not predict the results of those players who join later to the competition.

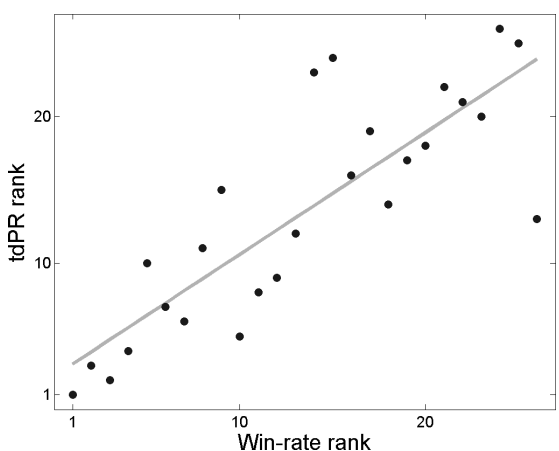

(a)

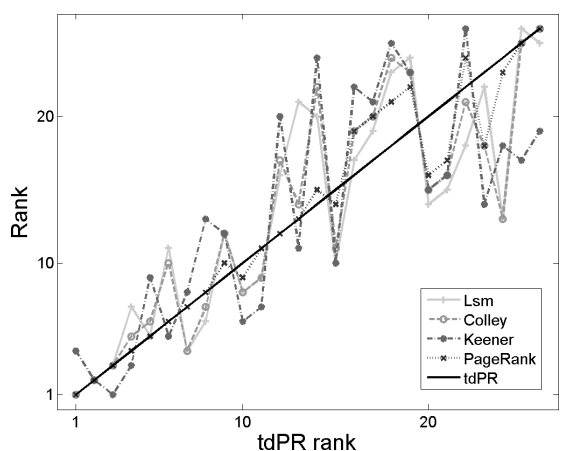

(b)

Figure 1: (a) The scatter plot of the tdPR rank vs. the win-rate rank. (b) The results obtained by the different ranking methods. 
Table 1: Scrores obtained by the different methods; the ordering of the players are obtained by the decreasing order of the tdPR values

\begin{tabular}{lllllllll}
\hline \hline Player & \#Plays & \#Wins & Win ratio & Lsm & Colley & Keener & PR & tdPR \\
\hline 9 & 13 & 13 & 1.000 & 1.418 & 1.074 & 0.229 & 0.113 & 0.138 \\
10 & 29 & 25 & 0.862 & 0.972 & 0.923 & 0.238 & 0.089 & 0.093 \\
12 & 30 & 26 & 0.867 & 0.859 & 0.882 & 0.245 & 0.083 & 0.085 \\
1 & 63 & 44 & 0.698 & 0.497 & 0.722 & 0.233 & 0.071 & 0.075 \\
14 & 6 & 3 & 0.500 & 0.658 & 0.717 & 0.198 & 0.064 & 0.070 \\
5 & 38 & 22 & 0.579 & 0.266 & 0.604 & 0.200 & 0.050 & 0.052 \\
23 & 5 & 3 & 0.600 & 0.779 & 0.736 & 0.199 & 0.047 & 0.047 \\
18 & 16 & 8 & 0.500 & 0.555 & 0.700 & 0.192 & 0.046 & 0.045 \\
11 & 24 & 11 & 0.458 & 0.209 & 0.564 & 0.193 & 0.039 & 0.040 \\
19 & 10 & 6 & 0.600 & 0.454 & 0.664 & 0.200 & 0.042 & 0.039 \\
21 & 13 & 7 & 0.538 & 0.325 & 0.615 & 0.199 & 0.035 & 0.032 \\
8 & 19 & 6 & 0.316 & -0.338 & 0.354 & 0.181 & 0.031 & 0.032 \\
26 & 1 & 0 & 0.000 & -0.503 & 0.407 & 0.194 & 0.031 & 0.029 \\
4 & 19 & 3 & 0.158 & -0.474 & 0.265 & 0.172 & 0.025 & 0.026 \\
6 & 10 & 5 & 0.500 & 0.269 & 0.586 & 0.194 & 0.030 & 0.025 \\
2 & 17 & 3 & 0.176 & -0.380 & 0.307 & 0.177 & 0.022 & 0.024 \\
17 & 13 & 2 & 0.154 & -0.437 & 0.286 & 0.178 & 0.019 & 0.020 \\
3 & 13 & 1 & 0.077 & -0.615 & 0.213 & 0.171 & 0.019 & 0.020 \\
7 & 12 & 2 & 0.167 & -0.650 & 0.219 & 0.176 & 0.018 & 0.018 \\
16 & 2 & 0 & 0.000 & -0.322 & 0.401 & 0.191 & 0.024 & 0.018 \\
13 & 2 & 0 & 0.000 & -0.322 & 0.401 & 0.191 & 0.024 & 0.018 \\
22 & 14 & 1 & 0.071 & -0.433 & 0.277 & 0.169 & 0.016 & 0.016 \\
24 & 4 & 1 & 0.250 & -0.507 & 0.349 & 0.191 & 0.023 & 0.016 \\
15 & 5 & 1 & 0.200 & -0.174 & 0.416 & 0.188 & 0.017 & 0.010 \\
25 & 3 & 0 & 0.000 & -1.060 & 0.186 & 0.191 & 0.015 & 0.007 \\
20 & 5 & 0 & 0.000 & -1.047 & 0.136 & 0.184 & 0.010 & 0.004 \\
\hline \hline
\end{tabular}

Table 2: Kendall's $\tau$ rank correlation between the different methods.

\begin{tabular}{lllllll}
\hline \hline & Win/loss & Lsm & Colley & Keener & PR & tdPR \\
Win/loss & 1.000 & & & & & \\
MASSEY & 0.705 & 1.000 & & & & \\
COLLEY & 0.748 & 0.895 & 1.000 & & & \\
KEENER & 0.655 & 0.606 & 0.711 & 1.000 & & \\
PR & 0.723 & 0.735 & 0.803 & 0.662 & 1.000 & \\
tdPR & 0.723 & 0.674 & 0.705 & 0.563 & 0.902 & 1.000 \\
\hline \hline
\end{tabular}




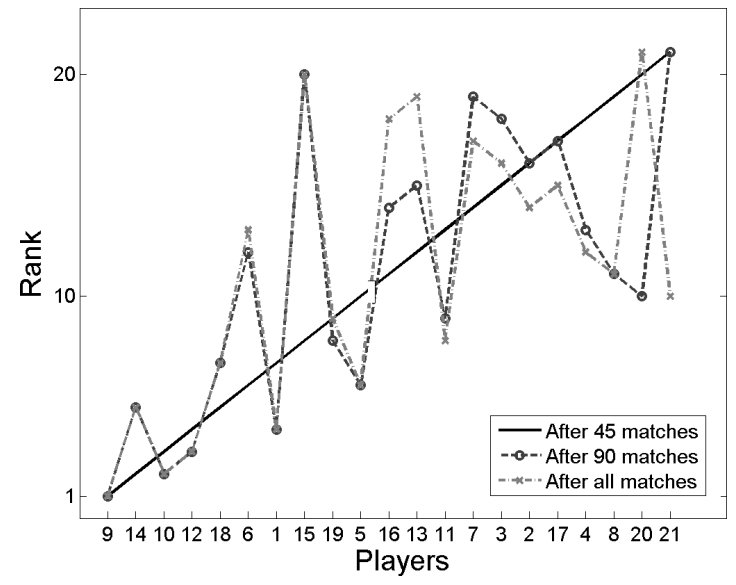

Figure 2: The tdPR ranks of the players after 45, 90 and 180 played games.

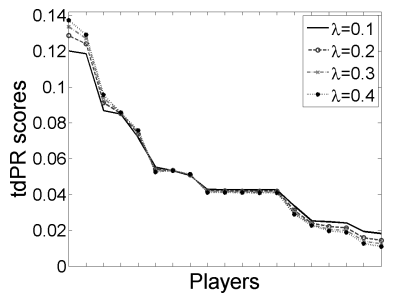

(a)

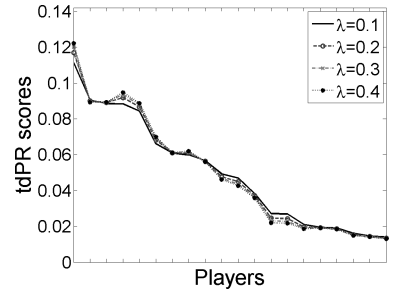

(b)

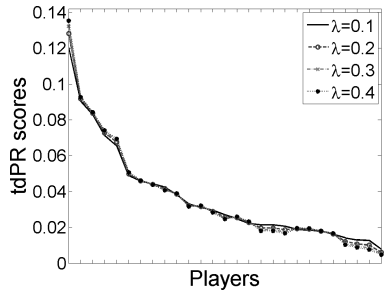

(c)

Figure 3: Sensitivity analysis of tdPR for different $\lambda$ values after 45,90 and 180 played games, from left to right. The figure shows, that the tdPR score is robust against these variations of $\lambda$.

\section{Further ideas and future work}

We also ran a clustering algorithm (aiming to maximize modularity [25]) to see whether there exists a deeper organizational mechanism behind the evolution of such a network. In Figure 2 the clusters are colored with different colors. Figure 4 illustrates the contact graph of the players after 90 played matches (left hand side) and the current state of the championship with more than 180 mathces (right hand side). It is interesting to see the changes of the clusters of the two graph. First, we observed that most of the new players wants to play against the actual best players (in tdPR rank) hoping to jump to the top of the ranking table. Second, it seems that players having closer tdPR values more likely to play with each other, than players having much less tdPR value and rank. Thus, we conjecture that the tdPR scores have a good explanatory power for a self-organizing mechanism of free-time sports and it can explain the appearance of different strength classes in most 

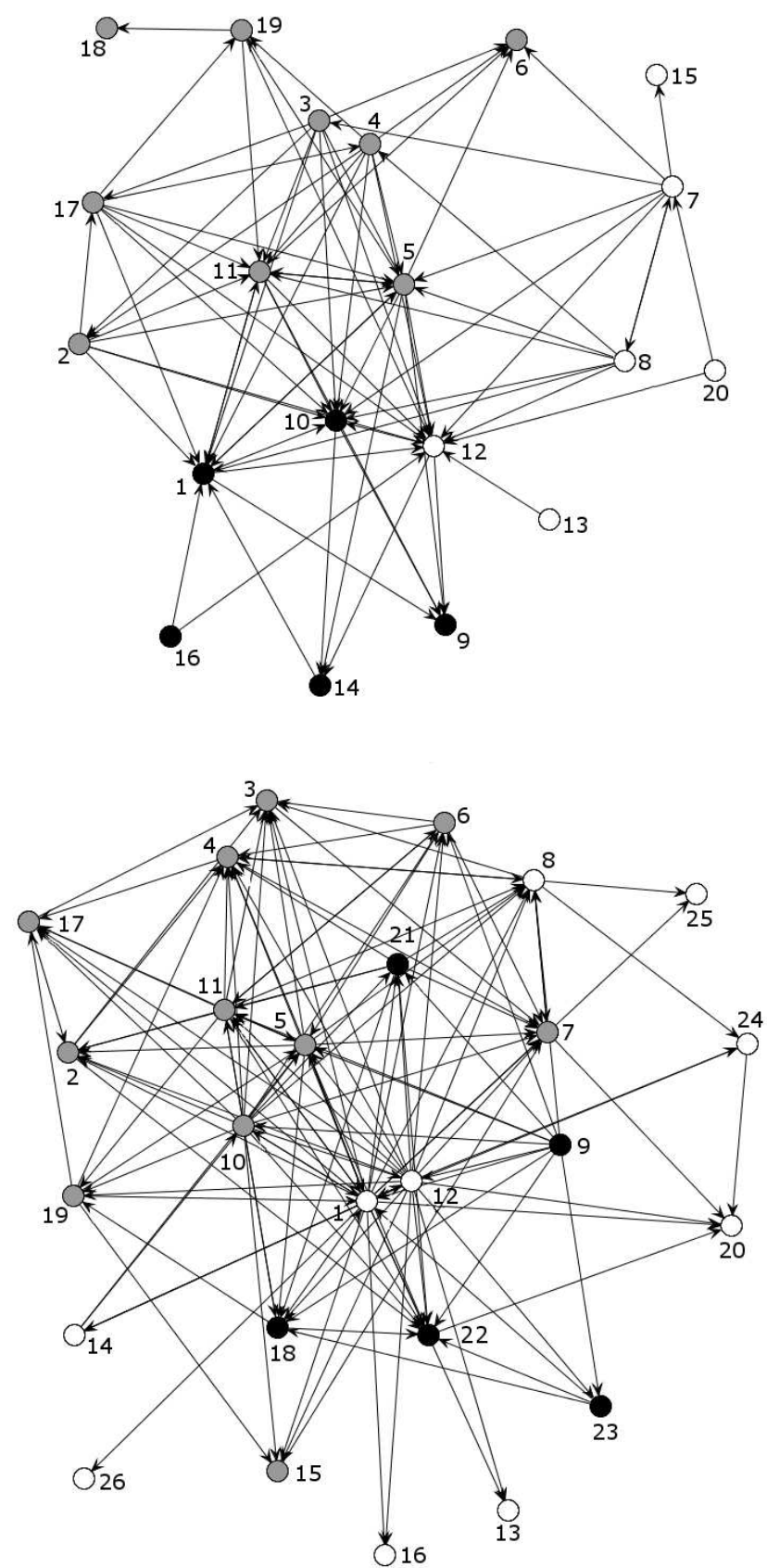

Figure 4: The contact graph of the players after 90 played matches (top) and the current state of the championship with more than 180 mathces (bottom). Nodes having same colors belong to the same clusters. 
of the sports, where the results in a class are more difficult to be predicted than results between different classes. Furthermore, in a graph theoretical point of view, a new type of 'regulatory' (for some details, see [9]) can be defined on directed graphs, where the fraction of in/out edges of a node is around $1 / 2$ in the same class, and tends to 1 (or 0 reversely) between different classes.

\section{Conclusions}

Graph based algorithms have been proved to be relevant in a wide range of applications. However there is no perfect algorithm for ranking sport players/teams, we believe that PageRank based methods are reliable to ranking athletes and this is even more true for time-dependent modifications of these stochastic algorithms.

In this work, we defined a time-dependent PageRank based algorithm and applied it for ranking players in a university table tennis competition. According to our tdPR method, the ranking of a player is not only determined by the number of his or her victories, but matters from how good players he could beat or lose against. It means, that a good player is needed to beat for higher ranking position, but win many matches against weaker opponents does not lead anyone to the first positions in the ranking table. The time-dependency of weights of the matches guarantee that the matches played a long time ago do not count as much weight in the ranking. Another aim of the time-dependency is to pressure the players to play regularly or else their results would be out of date, therefore count much less in the ranking.

We also observed that our method has a good predictive power. This can be interesting in other aspects of sports, for example estimate the betting odds for games. Finally, we think that a self-organization mechanism works in the background of the evolution of the contact graph. Obviously, players want to enter matches are expected to be exciting, but this nature of such competitions can be modeled and measured mathematically just by knowing the time-series of the results. That observation gives the idea to define a special preferential attachment mechanism [24] where players having higher PageRank values more likely to play (contact) with each other and this is maybe related to the emergence of an elite in sports. Further research is needed around this hypothesis, and testing our method for different sports and data sets is also another work for the future.

\section{Acknowledgment}

The first author was supported by the European Union and the State of Hungary, cofinanced by the European Social Fund in the framework of TÁMOP-4.2.4.A/2-11-12012-0001 'National Excellence Program'.

The authors also thank Tibor Csendes and András Pluhár for useful discussion and every excellent researcher of the Informatics Institute of University of Szeged who take time to relax at the ping-pong table. We are indebted to the referees for their careful reading and numerous suggestions which improved the presentation of the paper. 


\section{References}

[1] http://www.inf.u-szeged.hu/ london/TableTennisResults.txt.

[2] Baeza-Yates, Ricardo, Saint-Jean, Felipe, and Castillo, Carlos. Web structure, dynamics and page quality. String Processing and Information Retrieval, Lecture Notes in Computer Science, 2476:117-130, 2002.

[3] Bahmani, Bahman, Chowdhury, Abdur, and Goel, Ashish. Fast incremental and personalized pagerank. Proc. VLDB Endow., 4(3):173-184, December 2010.

[4] Berkhin, Pavel. A survey on PageRank computing. Internet Mathematics, 2(1):73120, 2005.

[5] Boginski, Vladimir, Butenko, Sergiy, and Pardalos, Panos M. Matrix-based methods for college football rankings. Economics, Management and Optimization in Sports, pages $1-13,2004$.

[6] Bradley, Ralph Allan and Terry, Milton E. Rank analysis of incomplete block designs: I. the method of paired comparisons. Biometrika, 39(3/4):324-345, 1952.

[7] Brin, Sergey and Page, Lawrence. The anatomy of a large-scale hypertextual web search engine. Computer networks and ISDN systems, 30(1):107-117, 1998.

[8] Colley, Wesley N. Colley's bias free college football ranking method: the Colley matrix explained. http://www. colleyrankings.com/matrate.pdf, 2002.

[9] Csaba, Béla and Pluhár, András. Weighted regularity lemma with applications. arXiv preprint arXiv:0907.0245, 2009.

[10] Csató, László. Ranking by pairwise comparisons for Swiss-system tournaments. Central European Journal of Operations Research, 21(4):783-803, 2013.

[11] Dahl, Geir. A matrix-based ranking method with application to tennis. Linear Algebra and its Applications, 437(1):26-36, 2012.

[12] David, Herbert A. Ranking from unbalanced paired-comparison data. Biometrika, 74(2):432-436, 1987.

[13] Govan, Anjela Yuryevna. Ranking theory with application to popular sports. PhD dissertation, North Carolina State University, Raleigh, North Carolina, 2008.

[14] Govan, Anjela Yuryevna, Langville, Amy N, and Meyer, Carl D. Offense-defense approach to ranking team sports. Journal of Quantitative Analysis in Sports, 5(1):119, 2009.

[15] Gulliksen, Harold. A least-squares solution for paired comparisons with incomplete data. Psychometrika, 21(2):125-134, 1956. 
[16] Jech, Thomas. The ranking of incomplete tournaments: A mathematician's guide to popular sports. The American Mathematical Monthly, 90(4):pp. 246-264+265-266, 1983.

[17] Keener, James P. The Perron-Frobenius theorem and the ranking of football teams. SIAM Review, 35(1):80-93, 1993.

[18] Kendall, Maurice G. A new measure of rank correlation. Biometrika, 30:81-93, 1938.

[19] Kendall, Maurice G and Babington Smith, B. On the method of paired comparisons. Biometrika, 31(3/4):324-345, 1940.

[20] Langville, Amy N and Meyer, Carl D. Deeper inside pagerank. Internet Mathematics, 1(3):335-380, 2004.

[21] Massey, Kenneth. Statistical models applied to the rating of sports teams. Master thesis, Bluefield College, 1997.

[22] Meyer, Carl D. Matrix analysis and applied linear algebra book and solutions manual, volume 2. Society for Industrial and Applied Mathematics, 2000.

[23] Motegi, Shun and Masuda, Naoki. A network-based dynamical ranking system for competitive sports. Scientific Reports, 2:904, 2012.

[24] Newman, Mark EJ. Clustering and preferential attachment in growing networks. Physical Review E, 64(2):025102, 2001.

[25] Newman, Mark EJ. Modularity and community structure in networks. Proceedings of the National Academy of Sciences of the USA, 103(23):8577-8582, 2006.

[26] Radicchi, Filippo. Who is the best player ever? A complex network analysis of the history of professional tennis. PloS ONE, 6(2):e17249, 2011.

[27] Ross, Sheldon M. Introduction to probability models. Academic Press, Ninth edition, 2007.

[28] Rossi, Ryan A and Gleich, David F. Dynamic pagerank using evolving teleportation. In Algorithms and Models for the Web Graph, Lecture Notes in Computer Science, volume 7323, pages 126-137. Springer, 2012.

[29] Sargolzaei, P and Soleymani, F. Pagerank problem, survey and future research directions. International Mathematical Forum, 5(19):937-956, 2010.

[30] Smith, John H. Adjusting baseball standings for strength of teams played. American Statistician, 10(3):23-24, 1956.

Received 13th November 2013 